\title{
Identification and characterization of miR-96, a potential biomarker of NSCLC, through bioinformatic analysis
}

\author{
TONGHUI CAI* , JIE LONG ${ }^{*}$, HONGYAN WANG, WANXIA LIU and YAJIE ZHANG \\ Department of Pathology, School of Basic Medical Science, Guangzhou Medical University, \\ Guangzhou, Guangdong 511436, P.R. China
}

Received November 8, 2016; Accepted June 6, 2017

DOI: $10.3892 /$ or.2017.5754

\begin{abstract}
Lung cancer is the leading cause of cancer-related death worldwide. The poor prognosis is partly due to lack of efficient methods for early diagnosis. MicroRNAs play roles in almost all aspects of cancer biology, and can be secreted into the circulation and serve as molecular biomarkers for the early diagnosis of cancer. In the present study, we determined the expression of miR-96 and the function of its target genes in lung cancer through bioinformatic analysis. Four microRNA expression profiles of lung cancer were downloaded from Gene Expression Omnibus and the data were analyzed using SPSS 16.0 software. Compared to the control group, expression of miR-96 was significantly increased in non-small cell lung cancer (NSCLC) (GSE51855), lung adenocarcinoma (GSE48414), stage I adenocarcinoma tissues (GSE63805) and the plasma of lung cancer patients (GSE68951). miR-96 was also elevated in six different NSCLC cell lines. However, the expression level of miR-96 was not related to the age, gender, clinical stage and histological subtype of the NSCLC patients. GO analysis of 78 predicted target genes of miR-96 showed that 42 of the obtained GO terms are highly associated with specific cellular processes including response to stimulus, signaling pathway, cell division, cell communication, cell migration and calcium signaling. KEGG results indicated that the miR-96 targets are mainly involved in the GnRH signaling pathway, long-term potentiation and insulin signaling pathway. In conclusion, miR-96, functioning as an oncogene, may play an important role in the development and progression of lung cancer. miR-96 may have the potential to serve as a molecular biomarker for the early diagnosis of NSCLC.
\end{abstract}

Correspondence to: Dr Yajie Zhang, Department of Pathology, School of Basic Medical Science, Guangzhou Medical University, Guangzhou, Guangdong 511436, P.R. China

E-mail: yajie.zhang@163.com

${ }^{*}$ Contributed equally

Key words: non-small cell lung cancer, miR-96, bioinformatics

\section{Introduction}

Based on GIOBALCAN, an estimated 1.8 million new lung cancer cases and 1.6 million deaths occurred in 2012. This makes lung cancer the leading cause of cancer-related death among males worldwide and females in developed countries (1). The poor prognosis is attributed to the lack of efficient methods for early diagnosis and lack of successful treatment for metastasis. Since non-small cell lung cancer (NSCLC), which accounts for approximately $85 \%$ of all lung cancer cases, is not very sensitive to chemotherapy and/or radiation, surgery remains the treatment of choice. However, most newly diagnosed NSCLC patients cannot undergo surgery due to local invasion or distant metastasis. Therefore, it is particularly important to study the molecular mechanisms underlying NSCLC, which may provide novel molecular targets for the early diagnosis of lung cancer.

MicroRNAs (miRNAs) are a group of non-coding RNAs ( $\sim 22$ nucleotides) that can degrade target mRNA transcripts directly or suppress their translation through complete or partial complementarity recognizing the 3'UTR of target mRNAs (2). miRNAs have been proven to play an important role in the post-transcriptional regulation of gene expression and are involved in almost all aspects of cancer biology such as tumor transformation, growth, angiogenesis and epithelialmesenchymal transition by inhibiting specific oncogenes or tumor-suppressor genes. Accumulating data indicate that miRNAs are present in body fluids including blood plasma and serum, urine, saliva and semen $(3,4)$ and circulating miRNA levels are more accurate than the protein-coding gene profiles in tumor typing (5). Therefore, miRNAs are more likely to be novel molecular biomarkers in the screening and monitoring of cancer patients (6).

In our previous study, we found that DAL-1 (differentially expressed in adenocarcinoma of the lung-1; also known as EPB41L3, 4.1B) has an important role in the invasion and metastasis of NSCLC (7). By using microRNA.org, TargetScan and PicTar, we predicted four miRNAs, miR-26a, miR-26b, miR-96 and miR-223, that regulate DAL-1. Data from several studies previously showed that miR-223 does not only promote the invasion of lung cancer cells but also the metastasis of gastric cancer via targeting tumor suppressor DAL-1 $(8,9)$. Our previous study demonstrated that both miR-26a and DAL-1 gene expression are decreased in NSCLC, and DAL-1 
is not a real target gene of miR-26a (10). Both miR-26b and miR-26a belong to the miR-26 family, and miR-26b has low expression levels in many types of cancer, such as epithelial ovarian (EOC) (11), hepatocellular carcinoma (HCC) (12), as well as colorectal cancer (13). In this study, we chose miR-96 as our research target.

MicroRNA-96 (hsa-miR-96, miR-96), located on chromosome 7 (7q31 34), belongs to the miR-183 gene family, which is the first gene cluster to be reported in the development and function of ciliated ectodermal cells and organs and is essential for the development and function of animal sensory organs $(14,15)$. With the growing interest in the miR-183 gene family, miR-96 has been detected to be highly expressed in various human tumors and involved in cancer development by regulating key genes in tumor cell division and apoptosis (16-18). Although studies have shown that miR-96 is overexpressed in lung cancer $(19,20)$, it still remains unclear whether miR-96 could be used for early diagnosis and how miR-96 affects the progression of lung cancer. Herein, we determined the expression of miR-96 and the function of its target genes in lung cancer through bioinformatic analysis, aiming to ascertain whether it is a potential molecular biomarker for the early diagnosis of NSCLC and to obtain clues for the pathogenesis of lung cancer.

\section{Materials and methods}

Affymetrix microarray. The microRNA expression profiles of lung cancer (GSE51855, GSE48414, GSE63805, GSE68951) were downloaded from Gene Expression Omnibus (http:// www.ncbi.nlm.nih.gov/geo/), which are based on the platform of Affymetrix Human Genome U133 Plus 2.0 Array.

Probe re-annotation. Four TEX texts (GPL7341, GPL16770, GPL18410, GPL16770) were downloaded from GEO public data platform, to find the probe number of the hsa-miR-96 gene in GSE51855, GSE48414, GSE63805, GSE68951, respectively.

Cell culture. The following cell lines were cultured individually in RPMI-1640 medium (Invitrogen; Thermo Fisher Scientific, Inc., Waltham, MA, USA): human lung adenocarcinoma (A549, NCI-H1299 and pAa), human lung large cell carcinoma (NCI-H460), human lung squamous cell carcinoma (NCI-H520), human lung small cell carcinoma (NCI-H446), human lung giant-cell carcinoma (95D) and human bronchial epithelial (16HBE) cell lines. The medium was supplemented with $10 \%$ fetal bovine serum (FBS; Gibco; Thermo Fisher Scientific, Inc.), $100 \mathrm{U} / \mathrm{ml}$ penicillin and $100 \mathrm{mg} / \mathrm{ml}$ streptomycin (Hyclone; GE Healthcare Life Sciences, Logan, UT, USA). Cells were maintained in $5 \% \mathrm{CO}_{2}$ at $37^{\circ} \mathrm{C}$.

Real-time quantitative PCR. Specific RT primers and TaqMan probe (American ABI Company) were used for quantitative detection of hsa-miR-96 (cat no. A25576) and reference gene U6 (cat no. 4426961). Total RNAs in cells were isolated using TRIzol reagent (Invitrogen, Carlsbad, CA, USA). The RNA yield and the ratio of absorbance at 260 to $280 \mathrm{~nm}$ (A260/ A280 ratio) were determined with the NanoDrop 2000 spectrophotometer (NanoDrop Technologies, Montchanin, DE,
USA). The cDNA synthesis and qRT-PCR were carried out using the TaqMan MicroRNA Reverse Transcription kit and TaqMan MicroRNA assays and TaqMan ${ }^{\circledR}$ Universal Master Mix, No AmpErase ${ }^{\circledR}$ UNG (all from ABI, USA), respectively, according to the manufacturer's protocol.qRT-PCR was carried out using Applied Biosystems ${ }^{\circledR} 7500$ real-time PCR systems (Applied Biosystems, Foster City, CA, USA). The experiment was repeated 3 times. The relative quantitative analysis was carried out using the $\Delta \Delta \mathrm{Ct}$ method and the control was used for normalization of miRNA expression.

Bioinformatic analysis of miR-96 target genes. The target genes of miR-96 were predicted using miRecords. The intersection prediction results from at least 6 miRNA target gene prediction databases were analyzed to reduce the false-positive rate. To explore the functional annotation and pathway enrichment of those predicted genes, the Gene Ontology (GO) and Kyoto Encyclopedia of Genes and Genomes (KEGG) database analyses were conducted using a Database for Annotation, Visualization and Integrated Discovery (DAVID) v6.7 online analysis tool with $\mathrm{P}<0.05$ as the significant threshold to obtain significant gene sets.

Statistical analysis. All data are presented as mean \pm SD and statistical analyses were processed using SPSS 16.0 statistical software. Wilcoxon's rank-sum test was used to compare the expression of miR-96 between lung cancer and normal lung tissues/plasma in GSE51855, GSE48414 and GSE68951. Wilcoxon matched-pairs signed ranks sum test was used to analyzed the miR-96 expression in GSE63805. Wilcoxon's rank-sum test and Kruskal-Wallis test were conducted to analyze the correlation of miR-96 expression with the clinicopathological features in GSE48414 and GSE51855. Independent-sample t-test was conducted to evaluate the miR-96 expression in lung cancer cell lines and 16HBE cells. A P-value of $<0.05$ was considered statistically significant.

\section{Results}

Expression of miR-96 in lung cancer tissues, plasma and cell lines. We analyzed four microRNA expression profiling datasets to explore the expression pattern of miR-96 in the tissues and plasma of lung cancer patients. The result indicate that, compared with the normal lung tissues, miR-96 was significantly increased in NSCLC (GSE51855, Fig. 1A and Table I, P<0.001), lung adenocarcinoma (GSE48414, Fig. 1B and Table II, P<0.001) and stage I adenocarcinoma tissues (GSE63805, Fig. 1C and Table III, $\mathrm{P}<0.001)$. In addition, the expression level of miR-96 in the plasma (GSE68951) of the lung cancer patients was significantly higher compared to that of the non-cancer lung disease patients (Fig. 1D and Table IV, $\mathrm{P}<0.05$ ).

We subsequently examined the level of miR-96 in different types of lung cancer cell lines and bronchial epithelial 16HBE cells using qRT-PCR. As shown in Fig. 2, the expression level of miR-96 was elevated in all the 6 NSCLC cell lines but downregulated in the small cell lung cancer NCI-H446 cells. The highest expression levels for miR-96 were found in squamous cell carcinoma NCI-H520, adenocarcinoma NCI-H1299 and pAa cells $(\mathrm{P}<0.001$ for each). 

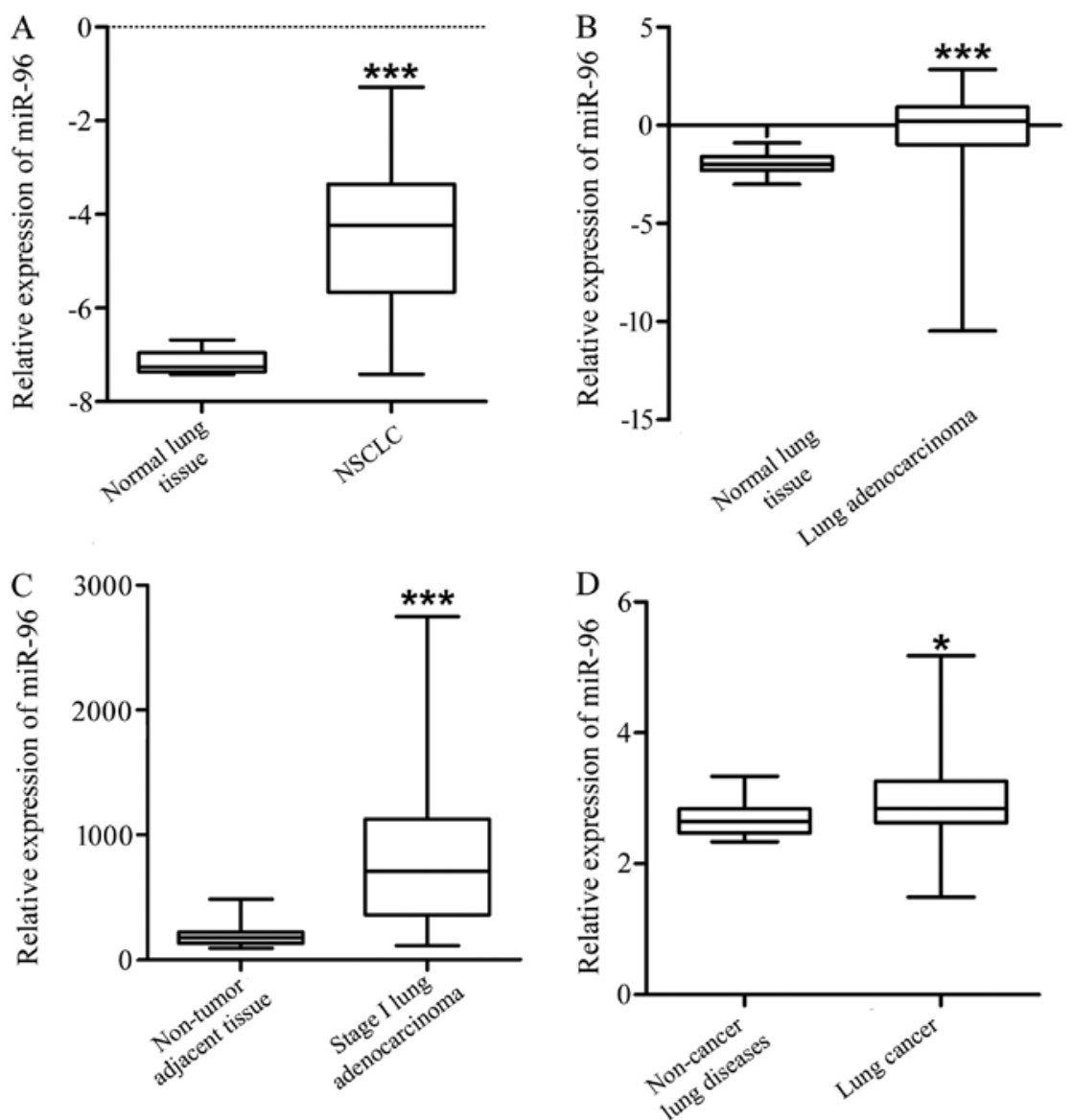

Figure 1. Expression of miR-96 in tissues and plasma from patients with lung cancer. (A) Dataset GSE51855 showed the miRNA profiling of 126 NSCLC and 5 normal lung tissues. miR-96 was overexpressed in the NSCLC tissues ( $\mathrm{P}=0.000)$. (B) Dataset GSE48414 showed the miRNA profiling of 154 lung adenocarcinoma and 20 paired normal lung tissues. miR-96 was overexpressed in the lung adenocarcinoma tissues $(\mathrm{P}=0.000)$. (C) Dataset GSE63805 showed the miRNA profiling of 32 stage I lung adenocarcinoma and 30 matched non-tumor adjacent tissues. miR- 96 was overexpressed in the stage I lung adenocarcinoma tissues $(\mathrm{P}=0.000)$. (D) Dataset GSE68951 showed the plasma miRNA profiling of 26 lung cancer and 12 non-cancer lung diseases. miR-96 was overexpressed in the plasma of the lung cancer patients $(\mathrm{P}=0.031)$. NSCLC, non-small cell lung cancer.

Table I. miR-96 expression in NSCLC and normal lung tissues (GSE51855).

\begin{tabular}{lrccc}
\hline Variables & $\mathrm{N}$ & Expression of miR-96 & $\chi^{2} / \mathrm{X}$ & P-value \\
\hline Total & 131 & & & \\
NSCLC & 126 & $-4.3623 \pm 0.12467$ & -3.699 & 0.000 \\
NA & 5 & $-7.1807 \pm 0.12815$ & & \\
\hline
\end{tabular}

NSCLC, non-small cell lung cancer; NA, normal lung tissues.

Table II. miR-96 expression in lung adenocarcinoma and normal lung tissues (GSE48414).

\begin{tabular}{lrrrr}
\hline Variables & $\mathrm{N}$ & Expression of miR-96 & $\chi^{2} / \mathrm{Z}$ & P-value \\
\hline Total & 174 & & & \\
ADC & 154 & $-0.548 \pm 0.12819$ & -5.804 & 0.000 \\
NA & 20 & $-1.9477 \pm 0.11705$ & & \\
\hline
\end{tabular}

NSCLC, non-small cell lung cancer; NA, normal lung tissues.
Table III. miR-96 expression in adenocarcinoma and adjacent non-tumor lung tissues (GSE63805).

\begin{tabular}{lllll}
\hline Variables & $\mathrm{N}$ & Expression of miR-96 & $\chi^{2} / \mathrm{Z}$ & P-value \\
\hline Total & 62 & & & \\
Stage I ADC & 32 & $849.71 \pm 114.63$ & -4.638 & 0.000 \\
NA & 30 & $184.93 \pm 13.41056$ & & \\
\hline
\end{tabular}

ADC, adenocarcinoma; NA, adjacent non-tumor lung tissues.

Table IV. miR-96 expression in the serum of NSCLC and non-cancerous pulmonary disease patients (GSE68951).

\begin{tabular}{lrrrr}
\hline Variables & $\mathrm{N}$ & Expression of miR-96 & $\chi^{2} / \mathrm{Z}$ & $\mathrm{P}$-value \\
\hline Total & 38 & & & \\
Lung cancer & 26 & $2.99409 \pm 0.0028$ & -2.159 & 0.031 \\
$\begin{array}{l}\text { Non-cancerous } \\
\text { lung diseases }\end{array}$ & 12 & $2.687812 \pm 0.0226$ & & \\
\hline
\end{tabular}

NSCLC, non-small cell lung cancer. 


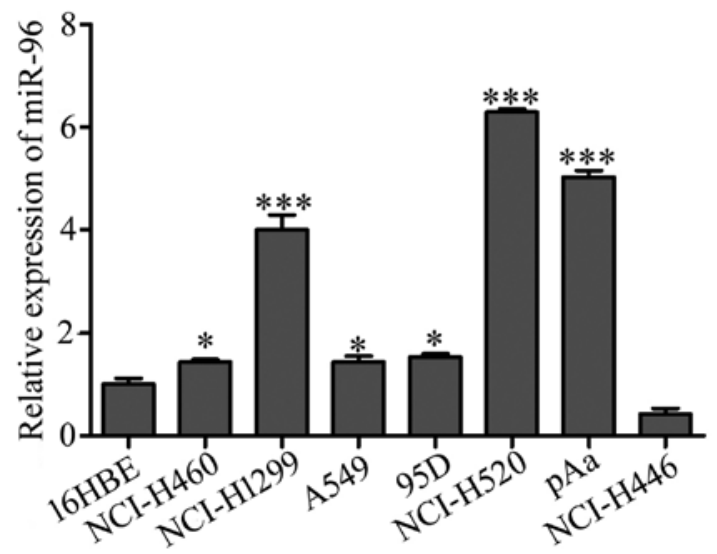

Figure 2. Expression of miR-96 in lung cancer cell lines. miR-96 expression in 7 lung cancer cell lines and human bronchinal epithelial 16HBE cells was determined by qRT-PCR. miR-96 was markedly increased in 6 NSCLC cell lines. ${ }^{*} \mathrm{P}<0.05,{ }^{* * *} \mathrm{P}<0.001$ vs. $16 \mathrm{HBE}$ cells.

Correlation between miR-96 expression and clinicopathological features of NSCLC. We then analyzed the correlation between miR-96expression and the clinicopathological features of NSCLC to further explore the potential role of miR-96 in the development and progression of lung cancer. Our results showed that the expression level of miR-96 in the tumors was not related to the age $(\mathrm{P}=0.631)$, sex $(\mathrm{P}=0.678)$, clinical stage $(\mathrm{P}=0.841)$ and histological subtype $(\mathrm{P}=0.051)$ of the NSCLC patients (GSE48414 and GSE51855, Tables V and VI).

\section{Bioinformation analysis of miR-96}

Prediction of miR-96 targets. Next, we used miRecords database to investigate miR-96 targets. miRecords does not only provide the target gene prediction of miRNAs but also the exact target genes regulated by miRNAs, which have already been experimentally validated. As shown in Table VII, a total of 71 target genes of miR-96 were predicted by at least six prediction softwares involved in miRecords. Ten miR-96 target genes were found and experimentally validated in the miRecords database, among which ADCY6, IRS1 and MYRIP were also in the prediction list. Finally, 71 predicted and 7 validated miR-96 targets (Table VII) were involved in the GO and KEGG analysis.

Gene ontology and KEGG pathway enrichment analysis of miR-96 target genes. Gene ontology enrichment analysis was performed to analyze 78 miR-96 target genes (Table VII). In total, $42 \mathrm{GO}$ terms were obtained, which included 24 biological processes, 15 cellular components and 3 molecular functions. These $42 \mathrm{GO}$ terms were sorted by P-values for further analysis and are listed in Tables VIII and IX.

Among the 24 biological process GO terms, the top 10 terms were: GO:0009725 (response to hormone stimulus), GO:0009719 (response to endogenous stimulus), GO:0010033 (response to organic substance), GO:0016197 (endosome transport), GO:0032228 (regulation of synaptic transmission, GABAergic), GO:0016055 (Wnt receptor signaling pathway), GO:0032868 (response to insulin stimulus), GO:0044057 (regulation of system process), GO:0007169 (transmembrane receptor protein tyrosine kinase signaling pathway) and GO:0042325 (regulation of phosphorylation) (Table VIII).
Table V. Correlation of miR-96 expression and the clinicopathological characteristics of the lung adenocarcinoma cases (GSE48414).

\begin{tabular}{lrrrr}
\hline Variables & $\mathrm{N}$ & Expression of miR-96 & $\chi^{2} / \mathrm{Z}$ & $\mathrm{P}$-value \\
\hline $\begin{array}{l}\text { Total } \\
\text { Age (years) }\end{array}$ & 154 & & & \\
$<65$ & 68 & $-0.0922 \pm 0.15804$ & -0.481 & 0.631 \\
$>65$ & 86 & $-0.0244 \pm 0.14538$ & & \\
Sex & & & & \\
$\quad$ Male & 67 & $0.0161 \pm 0.18784$ & -0.415 & 0.678 \\
$\quad$ Female & 87 & $-0.1094 \pm 0.12377$ & & \\
TNM & & & & \\
classification & & & & \\
$\quad$ I+II & 126 & $-0.069 \pm 0.14867$ & 0.04 & 0.841 \\
$\quad$ III+IV & 28 & $0.067259 \pm 0.04541$ & & \\
\hline
\end{tabular}

Table VI. Correlation of miR-96 expression and the different histological types of NSCLC (GSE51855).

\begin{tabular}{lrccc}
\hline Variables & $\mathrm{N}$ & Expression of miR-96 & $\chi^{2} / \mathrm{X}$ & P-value \\
\hline Total & 126 & & & \\
ADC & 76 & $-4.1497 \pm 0.15738$ & 7.75 & 0.051 \\
SQC & 29 & $-4.8942 \pm 0.21236$ & & \\
ASC & 4 & $-4.8262 \pm 0.74010$ & & \\
LCC & 17 & $-4.3103 \pm 0.40235$ & & \\
\hline
\end{tabular}

NSCLC, non-small cell lung cancer; ADC, adenocarcinoma; SQC, squamous cell carcinoma; ASC, adenosquamous carcinoma, LCC, large cell carcinoma.

The 15 cellular component GO terms were: GO:0042995 (cell projection), GO:0005815 (microtubule organizing center), GO:0005624 (membrane fraction), GO:0005626 (insoluble fraction), GO:0031095 (platelet dense tubular network membrane), GO:0005813 (centrosome), GO:0031094 (platelet dense tubular network), GO:0000267 (cell fraction), GO:0044463 (cell projection part), GO:0045202 (synapse), GO:0012505 (endomembrane system), GO:0005955 (calcineurin complex), GO: 0044430 (cytoskeletal part), GO:0045121 (membrane raft) and GO:0005856 (cytoskeleton) (Table IX).

In regards to the molecular function of the GO terms, GO:0005220 (inositol 1,4,5-trisphosphate-sensitive calcium-release channel activity), GO:0008095 (inositol-1,4,5trisphosphate receptor activity) and GO:0005516 (calmodulin binding) were the highest presented terms (Table X).

KEGG pathway analysis indicated that miR-96 target genes are mainly enriched in 9 pathways (Table XI). Among these pathways, hsa04912 (GnRH signaling pathway) (Fig. 3), hsa04114 (oocyte meiosis), hsa04720 (long-term potentiation) (Fig. 4), hsa04910 (insulin signaling pathway) (Fig. 5), hsa05215 (prostate cancer) and hsa04540 (gap junction) showed significantly higher enrichment, followed by hsa04916 
Table VII. The target genes of hsa-miR-96 investigated by miRecords database.

\begin{tabular}{|c|c|c|c|c|c|}
\hline No. & miRNA ID & Refseq & Symbol & Description & Note \\
\hline 1 & hsa-miR-96 & NM_015270 & ADCY6 & Adenylate cyclase 6 & Predicted \\
\hline 2 & hsa-miR-96 & NM_198715 & PTGER3 & Prostaglandin E receptor 3 (subtype EP3) & Predicted \\
\hline 3 & hsa-miR-96 & NM_016623 & FAM49B & Family with sequence similarity 49 , member B & Predicted \\
\hline 4 & hsa-miR-96 & NM_016565 & CHCHD8 & $\begin{array}{l}\text { Coiled-coil-helix-coiled-coil-helix } \\
\text { domain containing } 8\end{array}$ & Predicted \\
\hline 5 & hsa-miR-96 & NM_015516 & TSKU & Tsukushin & Predicted \\
\hline 6 & hsa-miR-96 & NM_015460 & MYRIP & Myosin VIIA and Rab interacting protein & Predicted \\
\hline 7 & hsa-miR-96 & NM_015215 & CAMTA1 & Calmodulin binding transcription activator 1 & Predicted \\
\hline 8 & hsa-miR-96 & NM_014946 & SPAST & Spastin & Predicted \\
\hline 9 & hsa-miR-96 & NM_014943 & ZHX2 & Zinc fingers and homeoboxes 2 & Predicted \\
\hline 10 & hsa-miR-96 & NM_014839 & LPPR4 & Plasticity related gene 1 & Predicted \\
\hline 11 & hsa-miR-96 & NM_012300 & FBXW11 & F-box and WD repeat domain containing 11 & Predicted \\
\hline 12 & hsa-miR-96 & NM_012257 & HBP1 & HMG-box transcription factor 1 & Predicted \\
\hline 13 & hsa-miR-96 & NM_007198 & PROSC & Proline synthetase co-transcribed homolog (bacterial) & Predicted \\
\hline 14 & hsa-miR-96 & NM_006940 & SOX5 & SRY (sex determining region Y)-box 5 & Predicted \\
\hline 15 & hsa-miR-96 & NM_006861 & RAB35 & RAB35, member RAS oncogene family & Predicted \\
\hline 16 & hsa-miR-96 & NM_006791 & MORF4L1 & Mortality factor 4 like 1 & Predicted \\
\hline 17 & hsa-miR-96 & NM_017974 & ATG16L1 & ATG16 autophagy related 16-like 1 (S. cerevisiae) & Predicted \\
\hline 18 & hsa-miR-96 & NM_018018 & SLC38A4 & Solute carrier family 38 , member 4 & Predicted \\
\hline 19 & hsa-miR-96 & NM_018243 & 11-Sep & Septin 11 & Predicted \\
\hline 20 & hsa-miR-96 & NM_198459 & DENND2C & DENN/MADD domain containing 2C & Predicted \\
\hline 21 & hsa-miR-96 & NM_194071 & CREB3L2 & cAMP responsive element binding protein 3-like 2 & Predicted \\
\hline 22 & hsa-miR-96 & NM_033505 & SELI & Selenoprotein I & Predicted \\
\hline 23 & hsa-miR-96 & NM_033260 & FOXQ1 & Forkhead box Q1 & Predicted \\
\hline 24 & hsa-miR-96 & NM_032560 & SMEK1 & SMEK homolog 1, suppressor of mek1 (Dictyostelium) & Predicted \\
\hline 25 & hsa-miR-96 & NM_032373 & PCGF5 & Polycomb group ring finger 5 & Predicted \\
\hline 26 & hsa-miR-96 & NM_032139 & ANKRD27 & Ankyrin repeat domain 27 (VPS9 domain) & Predicted \\
\hline 27 & hsa-miR-96 & NM_024915 & GRHL2 & Grainyhead-like 2 (Drosophila) & Predicted \\
\hline 28 & hsa-miR-96 & NM_024811 & FLJ12529 & Pre-mRNA cleavage factor I, $59 \mathrm{kDa}$ subunit & Predicted \\
\hline 29 & hsa-miR-96 & NM_022041 & GAN & Giant axonal neuropathy (gigaxonin) & Predicted \\
\hline 30 & hsa-miR-96 & NM_021229 & NTN4 & Netrin 4 & Predicted \\
\hline 31 & hsa-miR-96 & NM_020871 & $\mathrm{LRCH} 2$ & $\begin{array}{l}\text { Leucine-rich repeats and calponin } \\
\text { homology }(\mathrm{CH}) \text { domain containing } 2\end{array}$ & Predicted \\
\hline 32 & hsa-miR-96 & NM_020795 & NLGN2 & Neuroligin 2 & Predicted \\
\hline 33 & hsa-miR-96 & NM_020423 & SCYL3 & SCY1-like 3 (S. cerevisiae) & Predicted \\
\hline 34 & hsa-miR-96 & NM_020182 & PMEPA1 & Prostate transmembrane protein, androgen induced 1 & Predicted \\
\hline 35 & hsa-miR-96 & NM_006373 & VAT1 & $\begin{array}{l}\text { Vesicle amine transport protein } 1 \text { homolog } \\
(\text { T. californica) }\end{array}$ & Predicted \\
\hline 36 & hsa-miR-96 & NM_006283 & TACC1 & $\begin{array}{l}\text { Transforming, acidic coiled-coil } \\
\text { containing protein } 1\end{array}$ & Predicted \\
\hline 37 & hsa-miR-96 & NM_006016 & CD164 & CD164 molecule, sialomucin & Predicted \\
\hline 38 & hsa-miR-96 & NM_002959 & SORT1 & Sortilin 1 & Predicted \\
\hline 39 & hsa-miR-96 & NM_002923 & RGS2 & Regulator of G-protein signaling 2, $24 \mathrm{kDa}$ & Predicted \\
\hline 40 & hsa-miR-96 & NM_002833 & PTPN9 & Protein tyrosine phosphatase, non-receptor type 9 & Predicted \\
\hline 41 & hsa-miR-96 & NM_002734 & PRKAR1A & $\begin{array}{l}\text { Protein kinase, cAMP-dependent, regulatory, } \\
\text { type I, } \alpha \text { (tissue specific extinguisher } 1)\end{array}$ & Predicted \\
\hline 42 & hsa-miR-96 & NM_002515 & NOVA1 & Neuro-oncological ventral antigen 1 & Predicted \\
\hline 43 & hsa-miR-96 & NM_002265 & KPNB1 & Karyopherin (importin) $\beta 1$ & Predicted \\
\hline 44 & hsa-miR-96 & NM_002223 & ITPR2 & Inositol 1,4,5-triphosphate receptor, type 2 & Predicted \\
\hline 45 & hsa-miR-96 & NM_002222 & ITPR1 & Inositol 1,4,5-triphosphate receptor, type 1 & Predicted \\
\hline 46 & hsa-miR-96 & NM_002015 & FOXO1 & Forkhead box $\mathrm{O} 1$ & Predicted \\
\hline 47 & hsa-miR-96 & NM_001945 & HBEGF & Heparin-binding EGF-like growth factor & Predicted \\
\hline
\end{tabular}


Table VII. Continued.

\begin{tabular}{|c|c|c|c|c|c|}
\hline No. & miRNA ID & Refseq & Symbol & Description & Note \\
\hline 47 & hsa-miR-96 & NM_001945 & HBEGF & Heparin-binding EGF-like growth factor & Predicted \\
\hline 48 & hsa-miR-96 & NM_001931 & DLAT & Dihydrolipoamide S-acetyltransferase & Predicted \\
\hline 49 & hsa-miR-96 & NM_001839 & CNN3 & Calponin 3 , acidic & Predicted \\
\hline 50 & hsa-miR-96 & NM_000945 & PPP3R1 & $\begin{array}{l}\text { Protein phosphatase } 3 \text { (formerly } 2 B \text { ), regulatory } \\
\text { subunit } B, \alpha \text { isoform }\end{array}$ & Predicted \\
\hline 51 & hsa-miR-96 & NM_000935 & PLOD2 & Procollagen-lysine, 2-oxoglutarate 5-dioxygenase 2 & Predicted \\
\hline 52 & hsa-miR-96 & NM_000663 & ABAT & 4-aminobutyrate aminotransferase & Predicted \\
\hline 53 & hsa-miR-96 & NM_002998 & $\mathrm{SDC} 2$ & Syndecan 2 & Predicted \\
\hline 54 & hsa-miR-96 & NM_003060 & SLC22A5 & $\begin{array}{l}\text { Solute carrier family } 22 \\
\text { (organic cation/carnitine transporter), member } 5\end{array}$ & Predicted \\
\hline 55 & hsa-miR-96 & NM_003182 & $\mathrm{TAC} 1$ & Tachykinin, precursor 1 & Predicted \\
\hline 56 & hsa-miR-96 & NM_006007 & ZFAND5 & Zinc finger, AN1-type domain 5 & Predicted \\
\hline 57 & hsa-miR-96 & NM_005766 & FARP1 & $\begin{array}{l}\text { FERM, RhoGEF (ARHGEF) and pleckstrin } \\
\text { domain protein } 1 \text { (chondrocyte-derived) }\end{array}$ & Predicted \\
\hline 58 & hsa-miR-96 & NM_005544 & IRS 1 & Insulin receptor substrate 1 & Predicted \\
\hline 59 & hsa-miR-96 & NM_005502 & ABCA1 & ATP-binding cassette, sub-family A (ABC1), member 1 & Predicted \\
\hline 60 & hsa-miR-96 & NM_005400 & PRKCE & Protein kinase $\mathrm{C}, \varepsilon$ & Predicted \\
\hline 61 & hsa-miR-96 & NM_005277 & GPM6A & Glycoprotein M6A & Predicted \\
\hline 62 & hsa-miR-96 & NM_004985 & KRAS & v-Ki-ras2 Kirsten rat sarcoma viral oncogene homolog & Predicted \\
\hline 63 & hsa-miR-96 & NM_004958 & FRAP1 & FK506 binding protein 12-rapamycin associated protein 1 & Predicted \\
\hline 64 & hsa-miR-96 & NM_004926 & ZFP36L1 & Zinc finger protein $36, \mathrm{C} 3 \mathrm{H}$ type-like 1 & Predicted \\
\hline 65 & hsa-miR-96 & NM_004731 & SLC16A7 & $\begin{array}{l}\text { Solute carrier family } 16, \text { member } 7 \\
\text { (monocarboxylic acid transporter 2) }\end{array}$ & Predicted \\
\hline 66 & hsa-miR-96 & NM_004514 & FOXK2 & Forkhead box K2 & Predicted \\
\hline 67 & hsa-miR-96 & NM_004481 & GALNT2 & $\begin{array}{l}\text { UDP-N-acetyl- } \alpha \text {-D-galactosamine:polypeptide } \\
\text { N-acetylgalactosaminyltransferase } 2 \text { (GalNAc-T2) }\end{array}$ & Predicted \\
\hline 68 & hsa-miR-96 & NM_003654 & CHST1 & Carbohydrate (keratan sulfate Gal-6) sulfotransferase 1 & Predicted \\
\hline 69 & hsa-miR-96 & NM_003379 & EZR & Ezrin & Predicted \\
\hline 70 & hsa-miR-96 & NM_003342 & UBE2G1 & Ubiquitin-conjugating enzyme E2G 1 (UBC7 homolog, yeast) & Predicted \\
\hline 71 & hsa-miR-96 & NM_000332 & ATXN1 & Ataxin 1 & Predicted \\
\hline 72 & & NM_000863 & HTR1B & & Validated \\
\hline 73 & & NM_198159 & MITF & & Validated \\
\hline 74 & & NM_002015.3 & Foxo1 & & Validated \\
\hline 75 & & NM_0016513 & AQp5 & & Validated \\
\hline 76 & & NM_001408 & CELSR2 & & Validated \\
\hline 77 & & NM_153437 & ODF2 & & Validated \\
\hline 78 & & NM_001005861 & RYK & & Validated \\
\hline
\end{tabular}

(melanogenesis), hsa04270 (vascular smooth muscle contraction) and hsa04930 (Type II diabetes mellitus).

\section{Discussion}

Owing to its elevated expression, much effort has been dedicated to study the role of miR-96 in various types of cancers (21-23). In the majority of the tumors, miR-96 acts as an oncogene to promote the proliferation and invasion of cancer cells by inhibiting transcription factor FOXO1 (24), FOXO3a (25), tumor suppressor protein RECK (26) and metastasis suppressor protein MTSS1 (27). However, in pancreatic cancer, miR-96 functions as a tumor suppressor by targeting HERG1 and NUAK1 $(28,29)$.

There is no explicit conclusion whether miR-96 could affect the development and progression of lung cancer and serve as a molecular biomarker for the clinical diagnosis of lung cancer. By analyzing four microRNA expression profiles and qRT-PCR, we showed that miR-96 was markedly increased in NSCLC, lung adenocarcinoma, stage I adenocarcinoma tissues and NSCLC cell lines. Consistent with our result, Ma et al reported that miR-96 was significantly upregulated in six NSCLC tissues and its expression was then validated in an independent set of 35 pairs of tumors and their 
Table VIII. GO gene function (biological_process) analysis of the miR-96 targets.

\begin{tabular}{|c|c|c|c|c|}
\hline GO ID & GO ontology & GO term & Counts & P-value \\
\hline GO:0009725 & Biological_process & Response to hormone stimulus & 9 & 3.95E-04 \\
\hline GO:0009719 & Biological_process & Response to endogenous stimulus & 9 & 7.57E-04 \\
\hline GO:0010033 & Biological_process & Response to organic substance & 11 & 0.002442 \\
\hline GO:0016197 & Biological_process & Endosome transport & 4 & 0.002826 \\
\hline GO:0032228 & Biological_process & Regulation of synaptic transmission, GABAergic & 3 & 0.003041 \\
\hline GO:0016055 & Biological_process & Wnt receptor signaling pathway & 5 & 0.004019 \\
\hline GO:0032868 & Biological_process & Response to insulin stimulus & 4 & 0.012812 \\
\hline GO:0044057 & Biological_process & Regulation of system process & 6 & 0.0173 \\
\hline GO:0007169 & Biological_process & $\begin{array}{l}\text { Transmembrane receptor protein tyrosine kinase } \\
\text { signaling pathway }\end{array}$ & 5 & 0.023729 \\
\hline GO:0042325 & Biological_process & Regulation of phosphorylation & 7 & 0.025784 \\
\hline GO:0032870 & Biological_process & Cellular response to hormone stimulus & 4 & 0.027124 \\
\hline GO:0001666 & Biological_process & Response to hypoxia & 4 & 0.027651 \\
\hline GO:0043279 & Biological_process & Response to alkaloid & 3 & 0.028506 \\
\hline GO:0050804 & Biological_process & Regulation of synaptic transmission & 4 & 0.028721 \\
\hline GO:0051174 & Biological_process & Regulation of phosphorus metabolic process & 7 & 0.030557 \\
\hline GO:0019220 & Biological_process & Regulation of phosphate metabolic process & 7 & 0.030557 \\
\hline GO:0070482 & Biological_process & Response to oxygen levels & 4 & 0.03149 \\
\hline GO:0010648 & Biological_process & Negative regulation of cell communication & 5 & 0.032803 \\
\hline GO:0007612 & Biological_process & Learning & 3 & 0.03461 \\
\hline GO:0051969 & Biological_process & Regulation of transmission of nerve impulse & 4 & 0.034993 \\
\hline GO:0031998 & Biological_process & Regulation of fatty acid beta-oxidation & 2 & 0.03838 \\
\hline GO:0031644 & Biological_process & Regulation of neurological system process & 4 & 0.038689 \\
\hline GO:0043434 & Biological_process & Response to peptide hormone stimulus & 4 & 0.039324 \\
\hline GO:0046907 & Biological_process & Intracellular transport & 8 & 0.040338 \\
\hline
\end{tabular}

GO, Gene Ontology.

Table IX. GO gene function (cellular_component) analysis of the miR-96 targets.

\begin{tabular}{|c|c|c|c|c|}
\hline GO ID & GO ontology & Term & Count & P-value \\
\hline GO:0042995 & Cellular_component & Cell projection & 11 & $9.15 \mathrm{E}-04$ \\
\hline GO:0005815 & Cellular_component & Microtubule organizing center & 6 & 0.005289516 \\
\hline GO:0005624 & Cellular_component & Membrane fraction & 10 & 0.009055844 \\
\hline GO:0005626 & Cellular_component & Insoluble fraction & 10 & 0.011347551 \\
\hline GO:0031095 & Cellular_component & Platelet dense tubular network membrane & 2 & 0.013319656 \\
\hline GO:0005813 & Cellular_component & Centrosome & 5 & 0.017582227 \\
\hline GO:0031094 & Cellular_component & Platelet dense tubular network & 2 & 0.017720687 \\
\hline GO:0000267 & Cellular_component & Cell fraction & 11 & 0.020267702 \\
\hline GO:0044463 & Cellular_component & Cell projection part & 5 & 0.02029103 \\
\hline GO:0045202 & Cellular_component & Synapse & 6 & 0.020676761 \\
\hline GO:0012505 & Cellular_component & Endomembrane system & 9 & 0.021888271 \\
\hline GO:0005955 & Cellular_component & Calcineurin complex & 2 & 0.022102431 \\
\hline GO:0044430 & Cellular_component & Cytoskeletal part & 10 & 0.024057867 \\
\hline GO:0045121 & Cellular_component & Membrane raft & 4 & 0.025855924 \\
\hline GO:0005856 & Cellular_component & Cytoskeleton & 12 & 0.039405823 \\
\hline
\end{tabular}

GO, Gene Ontology. 
Table X. GO gene function (molecular_function) analysis of the miR-96 targets.

\begin{tabular}{|c|c|c|c|c|}
\hline GO ID & GO ontology & Term & Count & P-value \\
\hline GO:0005220 & Molecular_function & $\begin{array}{l}\text { Inositol 1,4,5-trisphosphate-sensitive calcium-release channel } \\
\text { activity }\end{array}$ & 2 & 0.01449 \\
\hline GO:0008095 & Molecular_function & Inositol-1,4,5-trisphosphate receptor activity & 2 & 0.01927 \\
\hline GO:0005516 & Molecular_function & Calmodulin binding & 4 & 0.03047 \\
\hline
\end{tabular}

GO, Gene Ontology.

Table XI. Pathways enrichment analysis of the miR-96 tagets (KEGG).

\begin{tabular}{|c|c|c|c|c|}
\hline GO ID & Name of pathway & Count & P-value & Genes \\
\hline hsa04912 & GnRH signaling pathway & 5 & 0.001862149 & $\begin{array}{l}\text { NM_004985, NM_002223, NM_002222, NM_015270, } \\
\text { NM_001945 }\end{array}$ \\
\hline hsa04114 & Oocyte meiosis & 5 & 0.002843987 & $\begin{array}{l}\text { NM_012300, NM_002223, NM_002222, NM_015270, } \\
\text { NM_000945 }\end{array}$ \\
\hline hsa04720 & Long-term potentiation & 4 & 0.005899778 & NM_004985, NM_002223, NM_002222, NM_000945 \\
\hline hsa04910 & Insulin signaling pathway & 5 & 0.005930538 & $\begin{array}{l}\text { NM_004958, NM_002015, NM_004985, NM_005544, } \\
\text { NM_002734 }\end{array}$ \\
\hline hsa05215 & Prostate cancer & 4 & 0.01237797 & NM_004958, NM_002015, NM_004985, NM_194071 \\
\hline hsa04540 & Gap junction & 4 & 0.01237797 & NM_004985, NM_002223, NM_002222, NM_015270 \\
\hline hsa04916 & Melanogenesis & 4 & 0.016481296 & NM_004985, NM_015270, NM_198159, NM_194071 \\
\hline hsa04270 & $\begin{array}{l}\text { Vascular smooth muscle } \\
\text { contraction }\end{array}$ & 4 & 0.02283774 & NM_002223, NM_002222, NM_015270, NM_005400 \\
\hline hsa04930 & Type II diabetes mellitus & 3 & 0.027134231 & NM_004958,NM_005544, NM_005400 \\
\hline
\end{tabular}

KEGG, Kyoto Encyclopedia of Genes and Genomes.

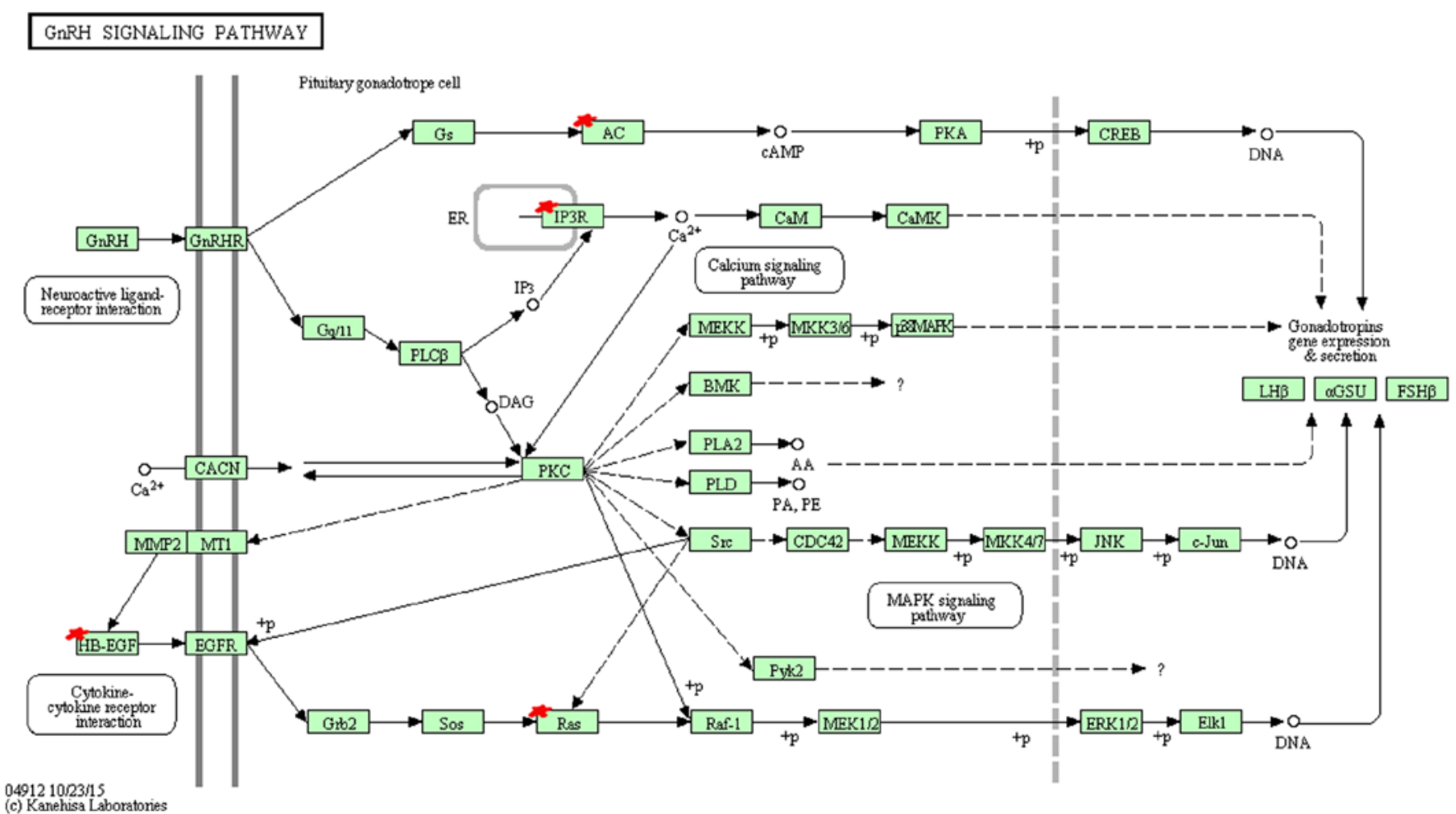

$0491210223 / 15$
(c) Kanehisa Laboratories

Figure 3. Five miR-96 target genes were enriched in the GnRH signaling pathway, including ADCY6 (AC), HBEGF, ITPR1 and ITPR2 (IP3R), and KRAS (Ras). 
LONG-TERM POTENTIATION

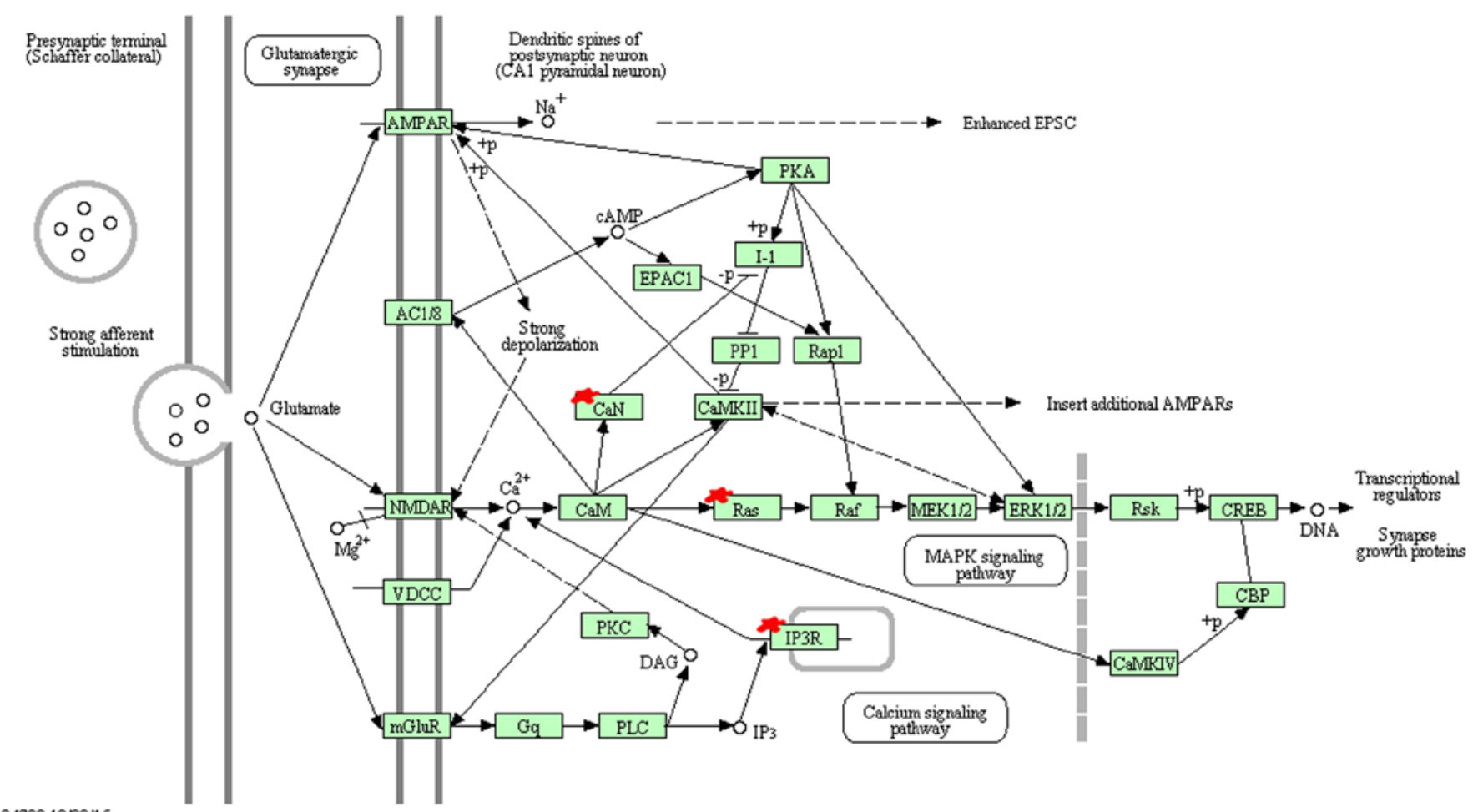

$0472010 / 23 / 15$

(c) Kanehisa Lahoratonies

Figure 4. Four miR-96 target genes were enriched in the long-term potentiation signaling pathway, including ITPR1 and ITPR2 (IP3R), PPP3R1 (CaN), KRAS (Ras).

INSULIN SIGNALING PATHWAY

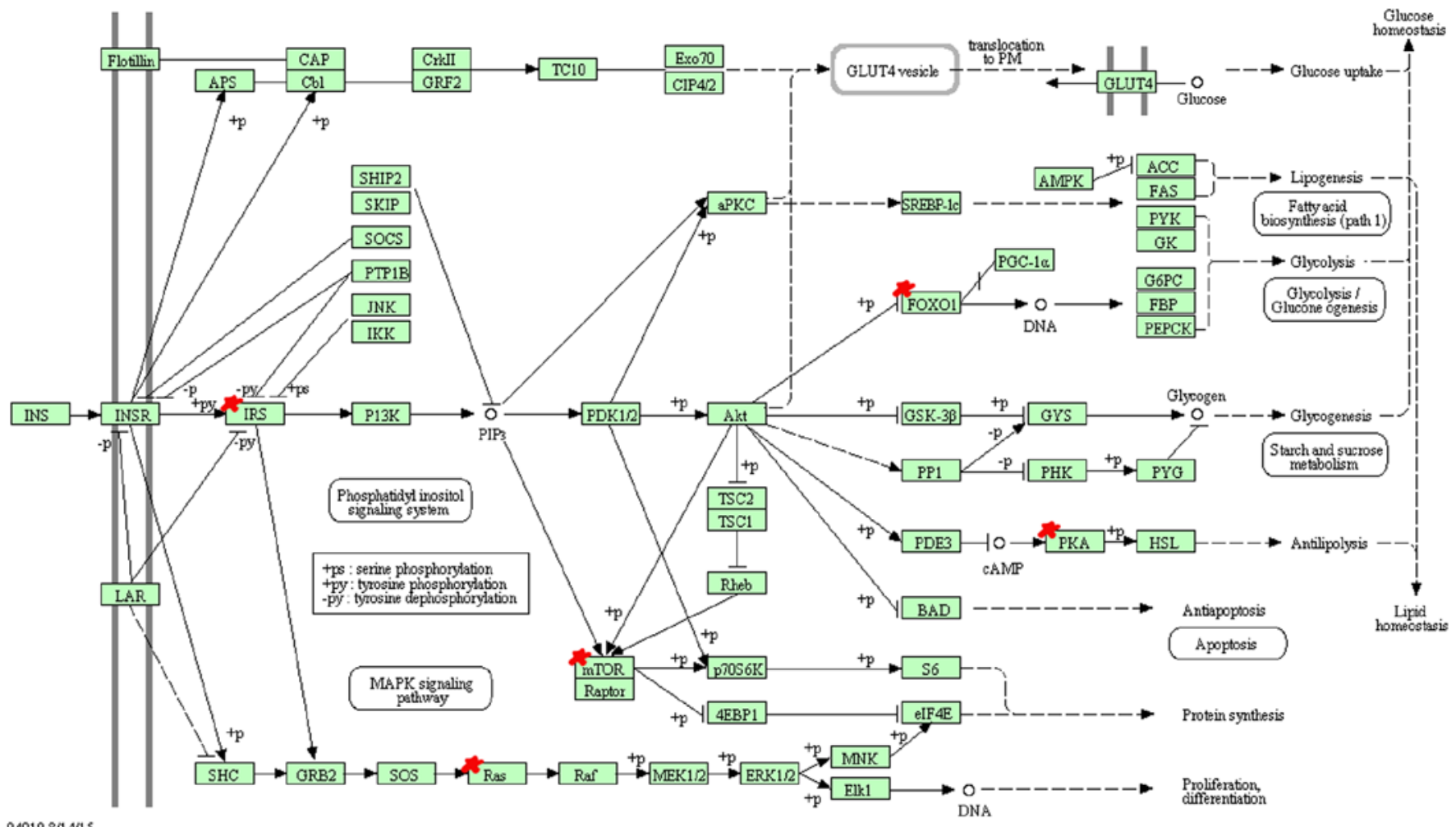
04910814415

Figure 5. Five miR-96 target genes were enriched in the insulin signaling pathway, including FOXO1, IRS1 (IRS), FRAP1 (mTOR), PRKAR1A (PKA) and KRAS (Ras). mTOR, mammalian target of rapamycin. 
adjacent normal tissues as well as in the serum of patients with NSCLC (19). To verify the microRNA expression signatures of lung cancer, Vosa et al performed a comprehensive metaanalysis of 20 published microRNA expression studies in lung cancer and identified a statistically significant microRNA meta-signature of seven upregulated microRNAs, including miR-21, miR-210, miR-182, miR-183, miR-31, miR-200b and miR-205. Since miR-182, miR-183 and miR-96 all belong to the miR-183 family, in conjunction with our results, miR-96 may serve as a novel molecular biomarker to distinguish early NSCLC patients from healthy individuals.

miRNAs are present not only in tissues but also in body fluids, such as blood, plasma, serum and sputum. Shen et al conducted several studies to assess the function of miRNAs in the sputum and plasma of lung cancer patients (30). They showed that the expression profile of plasma miR-21, miR-126, miR-210 and miR-486-5p produce high sensitivity and specificity in identifying stage I NSCLC patients. Zhu et al examined 70 pairs of lung cancer and non-cancerous tissues as well as serum samples. They found that miR-96 expression in tumors was positively associated with its expression in serum (31). Our data revealed that miR-96 expression in the plasma of lung cancer was significantly higher compared to that of non-cancer lung disease patients, suggesting that miR-96 may serve as a potential non-invasive marker for lung cancer diagnosis.

Although studies have shown that miR-96 is associated with poor overall survival in patients with pancreatic cancer (32), liver cancer (33) and colorectal cancer (34), our results did not demonstrate any significant correlation between the expression level of miR-96 and clinical stage as well as the histological subtype of the NSCLC patients. These discrepancies may be due to the different samples and databases that were used. Further studies are needed to confirm whether miR-96 could serve as a prognostic biomarker for lung cancer.

To date, computational methods have been widely used for the prediction of miRNAs and their target genes. However, the most commonly used miRNA target prediction websites, such as TargetScan, microRNA.org and PicTar, could not yield consistent results due to their different algorithm. miRecords is an integrated microRNA target database which includes a total of 11 established prediction programs. In this study, we selected the results predicted by at least six softwares in miRecords as the putative miR-96 target gene set and a collection of 78 predicted target genes were involved in GO/KEGG functional enrichment analysis. Since the GO hierarchy contains an added complexity by allowing terms to have multiple parents or ascendants, we used Fisher's exact 0.01 to reduce the redundancy in lists of enriched GO terms. Our data showed that among the 24 biological process GO terms obtained, the top 10 terms could be roughly grouped into several different categories including response to the stimulus (GO:0009725, GO:0009719, GO:0010033 and GO:0032868), signaling pathway (GO:0016055, GO:0032868, GO:0007169) and neurotransmission (GO:0032228). Tyrosine kinase signaling (GO:0007169) is currently known as the most successful molecular-targeted therapeutic approach for lung cancer (35). The canonical Wnt signaling pathway (GO:0016055), is another important regulator of proliferation (36) and metastasis (37) of non-small lung cancer cells. In addition, the 15 cellular component GO terms were significantly enriched in various specific processes with high frequency, such as cell division (GO:0005815, GO:0005813), cell communication (GO:0042995, GO:0044463) and cell migration (GO:0042995, GO:0044463, GO:0044430, GO:0005856), indicating that miR-96 may function as a regulator for the motility, migration and invasion of tumor cells. Moreover, three highly enriched molecular function GO terms (GO: 0005220, GO:0008095, and GO:0005516) suggest a potential new role of miR-96 in regulating calcium signaling important for tumor cell proliferation, apoptosis and migration.

In the KEGG annotation, GnRH signaling pathway (hsa04912), oocyte meiosis (hsa04114), long-term potentiation (hsa04720), insulin signaling pathway (hsa04910) and prostate cancer (hsa05215) showed the highest enrichment. GnRH has been reported to participate in the self-renewal of A549derived lung cancer stem-like cells by upregulating the JNK signaling pathway (38). Insulin, bound to insulin receptor, promotes cell proliferation through the RAS-RAF-MAP kinase signaling pathway and regulates cell survival process through (PI3K)-Akt-mammalian target of rapamycin (mTOR) pathway, playing an important role in the clinical treatment of NSCLC (39). Long-term potentiation and prostate cancer pathway, related to transcription regulation, cancer cell survival and proliferation respectively, suggest the potential function for miR-96 in cancer growth.

Although DAL-1 was not in the list of the 78 targets, DAL-1 was predicted as the target gene of miR-96 by 5 predicted databases of miRecords: MirTarget2, PicTar, PITA, RNAhybird, and TargetScan/TargetScanS (data not shown). For future studies, comprehensive screening, confirmation experiments and further bioinformatic analysis using available web tools such as Ingenuity Pathway Analysis (IPA) and STRINGProtein-Protein Interaction Networks need to be carried out on the predicted targets to explore the novel regulatory mechanism of miR-96 in cancer metastasis.

In conclusion, our results showed that miR-96, functioning as an oncogene, may play an important role in the development and progression of lung cancer. Both in tissue and plasma, miR-96 may have the potential to serve as a molecular biomarker for the early diagnosis of NSCLC.

\section{Acknowledgements}

This study was funded by the National Nature Science Foundation of China (no.81401391),Ph.D.Programs Foundation of the Ministry of Education of China (no. 20134423110001); National Nature Science Foundation of Guangdong Province (no.S2012010010181); Science and Technology Project of Guangzhou City (no. 2014Y2-00171) and Education System Innovative Academic Team of Guangzhou City (no. 13C06); Guangzhou City-Belonged Universities Scientific Research Program (no. 2012C130); National Natural Science Foundation of Guangdong Province (no. 2015A030313452).

\section{References}

1. Torre LA, Bray F, Siegel RL, Ferlay J, Lortet-Tieulent J and Jemal A: Global cancer statistics, 2012. CA Cancer J Clin 65: 87-108, 2015 
2. Conte I, Banfi S and Bovolenta P: Non-coding RNAs in the development of sensory organs and related diseases. Cell Mol Life Sci 70: 4141-4155, 2013.

3. Mitchell PS, Parkin RK, Kroh EM, Fritz BR, Wyman SK, Pogosova-Agadjanyan EL, Peterson A, Noteboom J, O'Briant KC, Allen A, et al: Circulating microRNAs as stable blood-based markers for cancer detection. Proc Natl Acad Sci USA 105: 10513-10518, 2008.

4. Weber JA, Baxter DH, Zhang S, Huang DY, Huang KH, Lee MJ, Galas DJ and Wang K: The microRNA spectrum in 12 body fluids. Clin Chem 56: 1733-1741, 2010.

5. Boutros PC, Lau SK, Pintilie M, Liu N, Shepherd FA, Der SD, Tsao MS, Penn LZ and Jurisica I: Prognostic gene signatures for non-small-cell lung cancer. Proc Natl Acad Sci USA 106 2824-2828, 2009.

6. Roepman P, Jassem J, Smit EF, Muley T, Niklinski J, van de Velde T, Witteveen AT, Rzyman W, Floore A, Burgers S, et al: An immune response enriched 72-gene prognostic profile for early-stage non-small-cell lung cancer. Clin Cancer Res 15: 284-290, 2009

7. Chen X, Guan X, Zhang H, Xie X, Wang H, Long J, Cai T, Li S, Liu Z and Zhang Y: DAL-1 attenuates epithelial-to mesenchymal transition in lung cancer. J Exp Clin Cancer Res 34: 3, 2015.

8. Liang H, Yan X, Pan Y, Wang Y, Wang N, Li L, Liu Y, Chen X, Zhang CY, Gu H, et al: MicroRNA-223 delivered by platelet-derived microvesicles promotes lung cancer cell invasion via targeting tumor suppressor EPB41L3. Mol Cancer 14: 58, 2015.

9. Li X, Zhang Y, Zhang H, Liu X, Gong T, Li M, Sun L, Ji G, Shi Y, Han Z, et al: miRNA-223 promotes gastric cancer invasion and metastasis by targeting tumor suppressor EPB41L3. Mol Cancer Res 9: 824-833, 2011.

10. Cai T, Guan X, Wang H, Fang Y, Long J, Xie X, et al: miR-26a regulates ANXA1, rather than DAL-1, in the development of lung cancer. Oncol Lett (accepted).

11. Lin J, Zhang L, Huang H, Huang Y, Huang L, Wang J, Huang S, He L, Zhou Y, Jia W, et al: miR-26b/KPNA2 axis inhibits epithelial ovarian carcinoma proliferation and metastasis through downregulating OCT4. Oncotarget 6: 23793-23806, 2015.

12. Shen G, Lin Y, Yang X, Zhang J, Xu Z and Jia H: MicroRNA-26b inhibits epithelial-mesenchymal transition in hepatocellular carcinoma by targeting USP9X. BMC Cancer 14: 393, 2014.

13. Zhang $\mathrm{C}$, Tong $\mathbf{J}$ and Huang G: Nicotinamide phosphoribosyl transferase (Nampt) is a target of microRNA-26b in colorectal cancer cells. PLoS One 8: e69963, 2013.

14. Pierce ML, Weston MD, Fritzsch B, Gabel HW, Ruvkun G and Soukup GA: MicroRNA-183 family conservation and ciliated neurosensory organ expression. Evol Dev 10: 106-113, 2008.

15. Kuhn S, Johnson SL, Furness DN, Chen J, Ingham N, Hilton JM, Steffes G, Lewis MA, Zampini V, Hackney CM, et al: miR-96 regulates the progression of differentiation in mammalian cochlear inner and outer hair cells. Proc Natl Acad Sci USA 108 : 2355-2360, 2011.

16. Guo $\mathrm{Y}$, Liu $\mathrm{H}$, Zhang $\mathrm{H}$, Shang $\mathrm{C}$ and Song $\mathrm{Y}$ : miR-96 regulates FOXO1-mediated cell apoptosis in bladder cancer. Oncol Lett 4 $561-565,2012$.

17. Yu N, Fu S, Liu Y, Xu Z, Liu Y, Hao J, Wang B and Zhang A: miR-96 suppresses renal cell carcinoma invasion via downregulation of Ezrin expression. J Exp Clin Cancer Res 34: 107, 2015.

18. Li C, Du X, Tai S, Zhong X, Wang Z, Hu Z, Zhang L, Kang P, Ji D, Jiang X, et al: GPC1 regulated by miR-96-5p, rather than miR-182-5p, in inhibition of pancreatic carcinoma cell proliferation. Int J Mol Sci 15: 6314-6327, 2014.

19. Ma L, Huang Y, Zhu W, Zhou S, Zhou J, Zeng F, Liu X, Zhang Y and $\mathrm{Yu} \mathrm{J}$ : An integrated analysis of miRNA and mRNA expressions in non-small cell lung cancers. PLoS One 6: e26502, 2011.

20. Guo H, Li Q, Li W, Zheng T, Zhao S and Liu Z: miR-96 downregulates RECK to promote growth and motility of non-small cell lung cancer cells. Mol Cell Biochem 390: 155-160, 2014.
21. Wu Z, Liu K, Wang Y, Xu Z, Meng J and Gu S: Upregulation of microRNA-96 and its oncogenic functions by targeting CDKN1A in bladder cancer. Cancer Cell Int 15: 107, 2015.

22. Fendler A, Jung M, Stephan C, Erbersdobler A, Jung K and Yousef GM: The antiapoptotic function of miR-96 in prostate cancer by inhibition of FOXO1. PLoS One 8: e80807, 2013.

23. Lin H, Dai T, Xiong H, Zhao X, Chen X, Yu C, Li J, Wang X and Song L: Unregulated miR-96 induces cell proliferation in human breast cancer by downregulating transcriptional factor FOXO3a. PLoS One 5: e15797, 2010.

24. Song HM, Luo Y, Li D-F, Wei CK, Hua KY, Song JL, Xu H, Maskey N and Fang L: MicroRNA-96 plays an oncogenic role by targeting FOXO1 and regulating AKT/FOXO1/Bim pathway in papillary thyroid carcinoma cells. Int J Clin Exp Pathol 8: 9889-9900, 2015

25. Gao F and Wang W: MicroRNA-96 promotes the proliferation of colorectal cancer cells and targets tumor protein p53 inducible nuclear protein 1, forkhead box protein O1 (FOXO1) and FOXO3a. Mol Med Rep 11: 1200-1206, 2015.

26. Zhang J, Kong X, Li J, Luo Q, Li X, Shen L, Chen L and Fang L: miR-96 promotes tumor proliferation and invasion by targeting RECK in breast cancer. Oncol Rep 31: 1357-1363, 2014.

27. Xu L, Zhong J, Guo B, Zhu Q, Liang H, Wen N, Yun W and Zhang L: miR-96 promotes the growth of prostate carcinoma cells by suppressing MTSS1. Tumour Biol 37: 12023-12032, 2016.

28. Feng J, Yu J, Pan X, Li Z, Chen Z, Zhang W, Wang B, Yang L, $\mathrm{Xu} \mathrm{H}$, Zhang $\mathrm{G}$, et al: HERG1 functions as an oncogene in pancreatic cancer and is downregulated by miR-96. Oncotarget 5 : 5832-5844, 2014.

29. Huang X, Lv W, Zhang JH and Lu DL: miR 96 functions as a tumor suppressor gene by targeting NUAK 1 in pancreatic cancer. Int J Mol Med 34: 1599-1605, 2014.

30. Shen J, Liu Z, Todd NW, Zhang H, Liao J, Yu L, Guarnera MA, Li R, Cai L, Zhan M, et al: Diagnosis of lung cancer in individuals with solitary pulmonary nodules by plasma microRNA biomarkers. BMC Cancer 11: 374, 2011.

31. Zhu W, Liu X, He J, Chen D, Hunag Y and Zhang YK: Overexpression of members of the microRNA-183 family is a risk factor for lung cancer: A case control study. BMC Cancer 11: 393, 2011.

32. Ebrahimi S, Hosseini M, Ghasemi F, Shahidsales S, Maftouh M, Akbarzade H, Parizadeh SA, Hassanian SM and Avan A: Circulating microRNAs as potential diagnostic, prognostic and therapeutic targets in pancreatic cancer. Curr Pharm Des 22: 6444-6450, 2016.

33. Leung WK, He M, Chan AW, Law PT and Wong N: Wnt/ $\beta$-catenin activates miR-183/96/182 expression in hepatocellular carcinoma that promotes cell invasion. Cancer Lett 362: 97-105, 2015.

34. Sun Y, Liu Y, Cogdell D, Calin GA, Sun B, Kopetz S, Hamilton SR and Zhang W: Examining plasma microRNA markers for colorectal cancer at different stages. Oncotarget 7: 11434-11449, 2016.

35. Kobayashi S, Boggon TJ, Dayaram T, Jänne PA, Kocher O, Meyerson M, Johnson BE, Eck MJ, Tenen DG and Halmos B: EGFR mutation and resistance of non-small-cell lung cancer to gefitinib. N Engl J Med 352: 786-792, 2005

36. Huang C, Ma R, Xu Y, Li N, Li Z, Yue J, Li H, Guo Y and Qi D: Wnt2 promotes non-small cell lung cancer progression by activating WNT/ $\beta$-catenin pathway. Am J Cancer Res 5: 1032-1046, 2015 .

37. Chen X, Song X, Yue W, Chen D, Yu J, Yao Z and Zhang L: Fibulin-5 inhibits Wnt/ $\beta$-catenin signaling in lung cancer. Oncotarget 6: 15022-15034, 2015.

38. Lu C, Huang T, Chen $\mathrm{W}$ and $\mathrm{Lu} \mathrm{H}$ : GnRH participates in the self-renewal of A549-derived lung cancer stem-like cells through upregulation of the JNK signaling pathway. Oncol Rep 34: 244-250, 2015

39. Scagliotti GV and Novello S: The role of the insulin-like growth factor signaling pathway in non-small cell lung cancer and other solid tumors. Cancer Treat Rev 38: 292-302, 2012. 